\title{
A Proposal for Combined Efforts Regarding Geophysical Research and Detection of Gravitational Waves
}

\author{
V. DF Sabrata - P. Fortini - C. Gunldi - S. Petralia (*)
}

Ricevuto il $2 s$ Febbraio 1970

SuMmary. - - The possibility of studying the Farth free oscillations with a laser interferometer is consiclered. In particular the problem of dotection of gravitational waves is analysed in tetail. This problem seems to be a very promising matter owing to the fact that with a two-mode He-No laser one may resolve changes in length down to $10^{-11} \mathrm{~cm}$. Other methods to reveal gravitational waves are mentioned.

Rrassunto. - Vengono prese in esame le possibilità oflerte da un interferometro laser per lo studio delle oscillazioni libere della terra. In particolare viene considerato il problema della rivelazione delle onde gravitazionali; la cosa sembra assai promettente sopratutto se si tiene conto che con un laser He-No a doppia frequenza, si jossono apprezzare spostamenti rell'ordine di $10^{-11} \mathrm{~cm}$. Vengono accennati anche altri possibili motodi di rivelazione delle onde gravitazionali.

Weber ( $\left.{ }^{1}\right)$ claims to have detected gravitational radiation in the $1660 \mathrm{~Hz}$ band; because of the extreme importance of this experiment and the significant cosmological implications, it is indispensable to check the existence of gravitational waves in different conditions and with different methods and techniques. Having this in mind we proposed at the beginning of 1968 to C.N.R. an outline of an experiment that, besides giving valuable informations on the soil preservation, allows us to study the Earth free oscillations and the possibility that some of these may be due to the interaction with gravitational radia-

(*) Istituto Nazionale di Fisica Nuclene - Sezione di Bologna lstituto di Fisica - Universitì di Bologna. 
tion. This project has been at last approved and we hope to start doing practically as soon as possible.

Our apparatus consists of a laser interferometer which allows to measure the soil deformations and which, in the ultimate model, should have an extent of the order of a kilometer. Is previously said, this instrument, besides observing the microseismic activity, the deformation of the earth crust, slower movements due to earthly tides and faulty movements, would be also utilized to measure free quadrupole oscillations of the Earth connected with the detection of gravitational waves. To discriminate from seismic waves one needs to accumulate data over a long interval of time and to look for Fourier components at the presumed source frequencies. Also the study of the decay time of the oscillations constitutes a tool to discriminate between waves of seismic and gravitational origin.

The instrument may be a He-Ne laser operating at a wave length of $6328 \AA$. With this kind of an instrument Vali and Bostrom (2,3,4) have been able to reach a sensitivity of $\Delta l / l \simeq 6 \cdot 10^{-12}$ and to reveal microseismic activity of a period of 6 sec and amplitude from 500 to $1500 \AA$.

The instrument noise on this type of measurements arises from:

a) optical path length changes by variation of vacuum in pipe, length change of pipe due to atmospheric pressure changes or temperature changes.

b) drift in the instrument piers.

c) laser frequency instability induced by changes of our FabryPerot reference cavity, as usual disposed for frequency stabilization.

d) fluctuations of the number of photons entering the detectors.

With a laser interferometer we can hope to measure extremely small strains $\varepsilon$ (where $\varepsilon=. J l l$ ) and we hope to increase the sensitivityof the instrument with the use of a double frequency laser. Recently Lichler and Wiesemann (5) have prospected the possibility of appraising a length shift of $10^{-3} \AA$ with a two-mode He-Ne laser in an optical resonator compled to the laser, and Herzinger and Lindner $\left(^{6}\right)$ have proposed a laser device to resolve changes in length down to $10^{-11} \mathrm{~cm}$.

Besides this we are planning to match the experiment with other experiments in coincidence, carried out with totally different methods.

Dyson ( ${ }^{7}$ ) gives an estimate of the displacement $\Delta l$ which would be measured on the Earth crust if the flux of incoming gravitational 
A PROPOSAL FOI COMHINED EFFUR'T REGARDIXg GEOPIYSICAL, ETC. 2:

waves, in the $1-\mathrm{Hz}$ frequency band. is of the order of

$$
F=\frac{C^{3} \omega^{2}|a|^{2}}{64 \pi G}=2 \cdot 10^{-5} \mathrm{erg} \operatorname{chl}^{-2} \sec ^{-1}
$$

He gives for the displacement $\Delta l$ :

$$
\Delta l \simeq 2 \cdot 10^{-17} \mathrm{cml} \text {. }
$$

This means that at the peak displacement the strain is

$$
\varepsilon \simeq 2 \frac{\Delta l}{\lambda}=10^{-22}
$$

$\lambda=\frac{C_{s}}{v}$ being the wave lenght connected with the sound velocity in Earth interior that is $C_{s}=4000 \mathrm{~m} / \mathrm{sec}$. This figure may be improved by many orders of magnitude: as a matter of fact we may take for the Earth a $Q$ of $\sim 400$ as given by geophysical estimates (see for instance Caputo $\left({ }^{8}\right)$ ) instead of $Q=1$ implied by Iyson's estimate, where $Q=E / \Delta E$ is the quality factor. Moreover the flux of gravitational waves on the $1-\mathrm{Hz}$ band may be greater than that assumed by Dyson. In fact, for instance, the flux revealed by Weber (1) in the $1-\mathrm{KHz}$ frequency band, that is

$$
F \sim 3 \cdot 10^{-1} \mathrm{erg} \mathrm{cm}^{-2} \sec ^{-1},
$$

is many order of magnitude greater than that foreseeable on the ground both of any known model of the Universe and of estimates which take into account possible sources of gravitational waves. If, for example, we assume a flux of $3 \cdot 10^{-1} \mathrm{erg} \mathrm{cm}^{-2} \mathrm{sec}^{-1}$ also in the $1-\mathrm{Hz}$ band, that is four order of magnitude greater than that assumed by Dyson, remembering that

$$
\varepsilon \sim C^{2} R^{i}{ }_{\text {oio }} Q / \omega^{2},
$$

where $R^{i}$ oio is the curvature tensor component which drives a gravitational wave detector and is proportional to the square root of the flux $R_{\text {oto }} \propto \sqrt{F}$, then the strain, with these new estimates of $Q$ and $F$, is

$$
\varepsilon \simeq 7 \cdot 10^{-18},
$$

and for the peak displacement we have:

$$
\Delta l \simeq 1.4 \cdot 10^{-12} \mathrm{~cm} .
$$

We think of improving further these figures using very large masses, well isolated from the Earth, and the laser as a transducer to 
detect their relative motion or, alternatively, the oscillations of a single large mass. In this case we may have a quality factor $Q \sim 10^{6}$ and the strain would be

$$
\varepsilon \sim 2 \cdot 10^{-14}
$$

with $\Delta l \simeq 4 \cdot 10^{-\theta} \mathrm{cm}$.

But, as we have said, the best would be to have some other device which, exploiting totally different principles, gives a measure of the gravitational waves flux: in this case we can look for coincidences between the two apparatus.

This new array will be founded on the interaction between gravitational waves and a static magnetic lield. Iupanov $\left(^{\circ}\right)$ has calculated the change of a static electric field of a capacitor in the field of a gravitational wave. We have made a calculation $\left({ }^{10}\right)$ along the same line, considering a magnetostatic field parallel to the direction of propagation of the gravitational wave ( $x$-direction). Between the surfaces of the polar expansions on the magneti we have a steady electromagnetic wave:

$$
E_{y}=-H_{z}=H^{(0)} x A \cos \delta \sin \delta e^{\left(x^{0}+\pi / 2\right)} \sin k x
$$

where $H^{(0)} x$ is the static magnetic field, $A$ is the amplitude of the incident gravitational wave, linked to the flux by:

$$
F=\frac{C^{3} \omega^{2}|A|^{2}}{64 \pi G}
$$

$\delta$ is the angle between the $x$-axis and the direction of oscillation of the source of the gravitational wave. The ratio between the change of the magnetic field and the static magnetic field, in the case of a flux $F \sim 3 \cdot 10^{-1} \mathrm{erg} \mathrm{cm}^{-2} \mathrm{sec}^{-1}$ is:

$$
\frac{H_{z}}{H^{(0)} x}=\frac{E_{y}}{H^{(0)} x}=10^{-19}
$$

The case of a gravitational wave interacting with a dipole magnetic field is being studied. This case seems to be promising to see if there is any relation between periodic micropulsations of the geomagnetic field and incident gravitational waves. 


\section{REFERENCES}

(1) Weber J., "Phys. Rev. Letters" 22, 1320 (1969).

(2) VAli V.-Bostrom R. C., " Earth and planetary science Letters 4, 436 (1968).

(3) VaLI V.-Bostrox R. C., "Nature", 220, Decemher 1968.

(1) VALi V.-Bostrom R. C., "Rev. of Scient. Instr." 39, 1304 (1968).

(5) Eichlfir H.-Wiesemaxi W., "Zeitschrift angew. Phys. ", 28, 129 (1969).

(') IIfrzinger G.-Lindner II., "Pliysics Letters", 24 A, 684 (1967).

(7) D)yson F. J., "Astroph. Journ.", 156, 529 (1969).

$\left.{ }^{8}\right)$ Cajuto M., "Elasticità e dissipazione", Zanichelli (1969).

$\left({ }^{9}\right)$ Jupanor C. A., "Zurn. Eksp. Teor. Fiz.", 52, 118 (1967); "Sov. Pliys. JETP ", 25, 76 (1967).

(10) Boccaletti D.-De Sabbata Y.-Fortini P.-Guaddi C., "Nuovo Cimento" (1970) in press. 